Quim. Nova, Vol. 35, No. 7, 1336-1342, 2012

\title{
CARACTERIZAÇÃO COMPOSICIONAL E TRANSESTERIFICAÇÃO DE ÓLEO DE MICROALGA: UMA ABORDAGEM COMPUTACIONAL
}

\author{
Rosana Coelho da Conceição*, Camila Viana Frasão, Sílvia Maria Cruzeiro da Silva, José Luiz de Medeiros, Ofélia de \\ Queiroz Fernandes Araújo e Marta Cristina Picardo
}

Departamento de Engenharia Química, Escola de Química, Universidade Federal do Rio de Janeiro, Av. Athos da Silveira Ramos, 149, CT, Bl. E, Ilha do Fundão, 21941-909 Rio de Janeiro - RJ, Brasil

Recebido em 24/8/11; aceito em 9/2/12; publicado na web em 15/6/12

\begin{abstract}
COMPOSITIONAL CHARACTERIZATION AND TRANSESTERIFICATION OF MICROALGAE OIL: A COMPUTATIONAL APPROACH. A procedure for compositional characterization of a microalgae oil is presented and applied to investigate a microalgae based biodiesel production process through process simulation. The methodology consists of: proposing a set of triacylglycerides (TAG) present in the oil; assuming an initial TAG composition and simulating the transesterification reaction (UNISIM Design, Honeywell) to obtain FAME characterization values (methyl ester composition); evaluating deviations of experimental from calculated values; minimizing the sum of squared deviations by a non-linear optimization algorithm, with TAG molar fractions as decision variables. Biodiesel from the characterized oil is compared to a rapeseed based biodiesel.
\end{abstract}

Keywords: biodiesel; compositional characterization; microalgae oil.

\section{INTRODUÇÃO}

Nas últimas décadas, verifica-se grande preocupação com as questões ambientais, acompanhada de demanda por novas rotas tecnológicas visando à redução de impactos ambientais associados ao desenvolvimento econômico. Dentre estes, destaca-se o elevado consumo de energia, majoritariamente de origem fóssil, com prognósticos de depleção de reservas. ${ }^{1-5}$ Este cenário promove a busca por fontes alternativas de energia para manter o desenvolvimento econômico e social, distingui-se o biodiesel por seu potencial de redução de impactos ambientais. ${ }^{6}$ Ressalta-se, contudo, que a diferença de custos de produção do diesel, sem impostos, e custos de oportunidade (valores pagos no mercado internacional para os óleos vegetais) indica o valor do subsídio a este combustível e é um fator relevante na decisão por alternativas econômicas ao diesel de petróleo. ${ }^{7}$ Adicionalmente, há de se considerar que as principais fontes de óleo para a produção de biodiesel são de culturas alimentares, incluindo soja e canola, ${ }^{8}$ estabelecendo um conflito entre as finalidades energética e alimentar no uso de terras aráveis. Outras limitações ao uso de biodiesel incluem restrições regionais e estruturais de mercado; alta demanda por água e fertilizantes ${ }^{9}$ e a necessária preservação da biodiversidade. ${ }^{10}$ Por último, a relação entre produção e consumo de energia deve ser favorável: enquanto para o álcool brasileiro esta relação é 8,3 , para o biodiesel de soja é estimada em apenas 1,43 .

A exploração de microalgas como fonte de óleos graxos pode atender a esses requisitos e, portanto, contribuir para complementar a demanda primária de biodiesel. ${ }^{11}$ Além do seu significativo potencial de sequestro de $\mathrm{CO}_{2}$, as microalgas são vantajosas frente ao cultivo de plantas oleaginosas, devido ao seu reduzido footprint (área ocupada pelo processo, sua "pegada ecológica"), resultante de alta taxa de crescimento, alto teor de lipídeo e pela possibilidade de cultivo em reatores. ${ }^{12,13}$ Esses organismos eucariotos são relativamente simples, não possuem tecidos (raízes, caules e folhas), podendo ser unicelulares ou pluricelulares (agregados de células formando colônias ou filamentos). Normalmente, são organismos fotoautotróficos e a presença de água se faz necessária no suporte físico, reprodução e difusão dos nutrientes. ${ }^{14,15}$

\footnotetext{
*e-mail: rosanaconceicao@eq.ufrj.br
}

A avaliação do uso de óleo de microalga como alternativa aos óleos vegetais na obtenção de biodiesel deve considerar as condições de processamento e as características do biodiesel obtido, e, para tal, a simulação de processos é uma ferramenta valiosa. Contudo, o uso de óleo de microalga em uma plataforma de simulação requer a sua caracterização composicional, meta complexa dada a grande quantidade de substâncias presentes em óleos vegetais. Este trabalho apresenta um procedimento de caracterização composicional de óleo de microalgas e o aplica na investigação de rota tecnológica para produção de biodiesel.

Os óleos vegetais são misturas complexas formadas, principalmente, por triglicerídeos (TAG), diglicerídeos (DAG), ácidos graxos livres (AGL) e fosfolipídios. ${ }^{5,16}$ Por representarem cerca de 90\% do óleo, os TAG's são os componentes mais importantes para a caracterização do óleo, além de serem os reagentes com o metanol na transesterificação para obtenção de biodiesel. ${ }^{16}$

As características físico-químicas do óleo dependem da identidade composicional dos triglicerídeos. Em especial, o grau de insaturação dos ácidos graxos exerce influência principalmente sobre o ponto de fusão da mistura, uma propriedade de interesse em combustíveis. ${ }^{17}$ De acordo com a norma de biodiesel publicada pela ASTM, o biocombustível produzido a partir de microalgas apresenta propriedades semelhantes às do biodiesel padrão, sendo mais estável de acordo com seu ponto de fulgor. ${ }^{3,18,19}$ A principal diferença entre óleos de microalgas e os óleos vegetais consiste no teor de ácidos graxos poli-insaturados, que são responsáveis pela oxidação do biodiesel, alteram as propriedades do produto e reduzem a sua aceitabilidade para uso na fabricação de biodiesel. ${ }^{20}$

\section{PARTE EXPERIMENTAL}

A metodologia desenvolvida teve como objetivo caracterizar a composição de TAG's no óleo de microalgas e baseia-se na proposta de um elenco de triacilglicerídios (TAG) capaz de representar óleos de microalgas e cálculo de propriedades do óleo transesterificado com simulador comercial (UNISIM Design, Honeywell). Estes óleos têm composições decorrentes da linhagem e das condições de crescimento. Consequentemente, a literatura tem uma variedade 
de informações composicionais, muitas expressas em lumps metabólicos (e.g., lipídios, proteínas e carbo-hidratos), e a maioria dos estudos de simulação de processos de transesterificação de óleos (vegetais e de microalgas) adota um único ácido graxo (a exemplo de Zhang et al. ${ }^{21}$ ).

O óleo empregado para exemplificar a metodologia foi definido a partir do elenco de ácidos graxos proposto por Lin et al. ${ }^{22}$ para uma linhagem de Isochrysis galbana. Esses autores empregaram 8 ácidos graxos e no presente estudo o elenco foi reduzido aos 5 principais - ácidos mirístico, palmítico, oleico, estearidônico e dodosa-hexaenoico, de acordo com a Tabela 1.

Tabela 1. Principais ácidos graxos encontrados na espécie Isochrysis galbana

\begin{tabular}{cccc}
\hline Símbolo & $\begin{array}{c}\text { Ácidos } \\
\text { Graxos }\end{array}$ & Nomes & $\begin{array}{c}\text { Composição } \\
\text { Normalizada }\end{array}$ \\
\hline M & $14: 00$ & Ácido Mirístico & 0,2619 \\
P & $16: 00$ & Ácido Palmítico & 0,2429 \\
O & $18: 1 \mathrm{n}-9$ & Ácido Oléico & 0,2024 \\
S & $18: 4 n-3$ & Ácido Estearidônico & 0,1667 \\
D & $22: 6 n-3$ & Ácido Dodosahexaenóico & 0,1262 \\
\hline
\end{tabular}

\section{Metodologia}

O procedimento de caracterização composicional do óleo é detalhado na Figura 1, e é composto por cinco etapas.

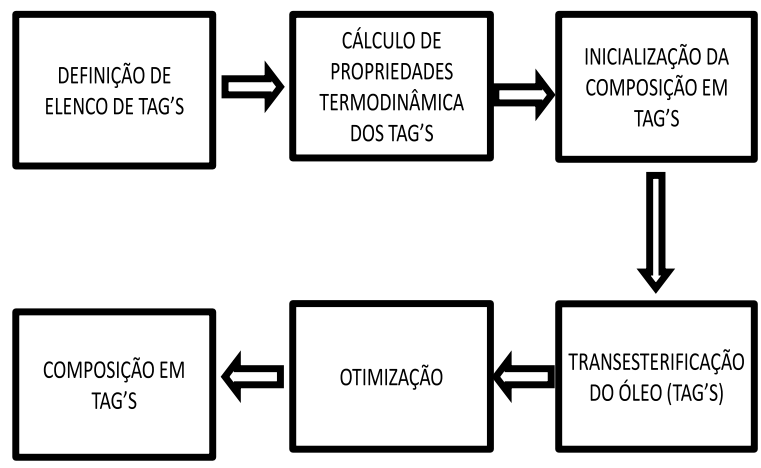

Figura 1. Representação das etapas para caracterização composicional do óleo de microalga

Etapa 1: Definição do elenco de triglicerídeos (TAG's)

Os triacilgliceróis (TAG), ou triglicerídeos, são ésteres formados a partir de ácidos graxos (AG) e glicerol (glicerina ou 1,2,3-propanotriol), com fórmula estrutural mostrada na Figura 2, onde R, R' e R", são cadeias alquílicas, podendo conter uma ou mais insaturações.<smiles>[R]C(=O)OCC(COC([R])=O)OC([R])=O</smiles>

Figura 2. Fórmula estrutural geral TAG's (ésteres de glicerina). Modificado da ref. 17

O número total de combinações possíveis dos cinco ácidos graxos da Tabela 1 (combinados três a três - R, R' e R) pode ser calculado conforme mostrado na Tabela 2, resultando em um conjunto de 35 triglicerídeos, sem a consideração de isômeros.
Tabela 2. Número de triglicerídeos versus número de ácidos graxos. Modificada da ref. 16

\begin{tabular}{cccc}
\hline \multirow{2}{*}{$\begin{array}{c}\text { Número de } \\
\text { Ácidos Graxos }\end{array}$} & \multicolumn{3}{c}{ Números de Triglicerídeos } \\
\cline { 2 - 4 } & Isômeros & $\begin{array}{c}\text { Sem isômeros } \\
\text { óticos }\end{array}$ & Sem isômeros \\
\hline $\mathrm{X}$ & $\mathrm{X}^{3}$ & $\left(\mathrm{X}^{3}+\mathrm{X}^{2}\right) / 2$ & $\left(\mathrm{X}^{3}+3 \mathrm{X}^{2}+2 \mathrm{X}\right) / 6$ \\
\hline 2 & 8 & 6 & 4 \\
3 & 27 & 18 & 10 \\
4 & 64 & 48 & 20 \\
5 & 125 & 75 & 35 \\
10 & 1000 & 550 & 220 \\
20 & 8000 & 4200 & 1540 \\
40 & 64000 & 32800 & 11480 \\
\hline
\end{tabular}

Etapa 2: Cálculo das propriedades termodinâmicas dos TAG's

$\mathrm{O}$ cálculo de propriedades cada TAG é realizado com o método de Constantinou e Gani ${ }^{23}$ (Equação 1).

$$
f(X)=\sum_{i} N_{i} C_{i}+w \sum_{j} M_{j} D_{j}+z \sum_{k} O_{k} E_{k}
$$

onde $C_{i}$ é a contribuição de grupo de primeira ordem, com frequência $N_{i} ; D_{j}$ é a contribuição de grupo de segunda ordem, com frequência $M_{j}$ e $E_{k}$ é a contribuição de grupo de terceira ordem, com frequência $O_{k}$. Os parâmetros $w$ e $z$ assumem valores nulos se não ocorrerem contribuições de segunda e terceira ordem, e 1 em caso contrário. A função $f(X)$ assume as expressões mostradas em Constantinou e Gani. ${ }^{23}$

Para cálculo do volume molar, utiliza-se a Equação 2,

$$
V_{1}-d=\sum_{i} N_{i} v_{1 i}+A \sum_{j} M_{j} v_{2 j}
$$

onde $v_{l i}$ é a contribuição de grupo de primeira ordem, com frequência $N_{i} ; v_{2 i}$ é a contribuição de grupo de segunda ordem com frequência $M_{j}$; $d$ é a constante universal igual a $0,01211 \mathrm{~m}^{3} \mathrm{kmol}^{-1}$ e $A$ assume o valor 0 se não houver contribuição do segundo grupo e o valor 1 se houver a contribuição deste grupo. ${ }^{22}$ Os valores utilizados neste trabalho para cada propriedade estão apresentados na Tabela $3 .^{23,24}$

Tabela 3. Contribuições dos grupos de $1^{\mathrm{a}}$ ordem e de $2^{\mathrm{a}}$ ordem do método de contribuição de grupos CG para obtenção das propriedades parciais dos TAG's. Combinação das refs. 23 e 24

\begin{tabular}{cccccc}
\hline $\begin{array}{c}\text { Grupo } 1^{\mathrm{a}} \\
\text { ordem }\end{array}$ & ${\mathrm{Tb} 1 \mathrm{i}^{23}}^{23}$ & $\mathrm{Tc}^{23}$ & $\mathrm{Pc}^{223}$ & $\mathrm{Vc} 1 \mathrm{i}^{23}$ & $\mathrm{v} 1 \mathrm{i}^{24}$ \\
\hline $\mathrm{CH}_{3}$ & 0,8491 & 1,7506 & 0,0186 & 68,35 & 0,0261 \\
$\mathrm{CH}_{2}$ & 0,7141 & 1,3327 & 0,0135 & 56,28 & 0,0164 \\
$\mathrm{CH}$ & 0,2925 & 0,596 & 0,0073 & 37,5 & 0,0071 \\
$\mathrm{CH}=\mathrm{CH}$ & 1,5596 & 3,0741 & 0,023 & 98,43 & 0,0269 \\
$\mathrm{CH}_{2} \mathrm{COO}$ & 2,985 & 5,9619 & 0,027 & 132,89 & 0,0357 \\
\hline $\begin{array}{c}\mathrm{Grupo} 2^{\mathrm{a}} \\
\text { ordem }\end{array}$ & $\mathrm{Tb} 2 \mathrm{j}$ & $\mathrm{Tc} 2 \mathrm{j}$ & $\mathrm{Pc} 2 \mathrm{j}$ & $\mathrm{Vc} 2 \mathrm{j}$ & $\mathrm{v} 2 \mathrm{j}$ \\
\hline $\begin{array}{c}\mathrm{CH} H_{2-} \\
-\mathrm{CH}=\mathrm{CH}\end{array}$ & 0,0537 & 0,0262 & 0,0008 & 0,14 & 0,0001 \\
\hline
\end{tabular}

O método apresenta bons resultados para temperaturas críticas de moléculas grandes, para as quais o valor da temperatura normal de ebulição não é conhecido experimentalmente. ${ }^{25}$ No procedimento empregado, as propriedades físicas estimadas pelo método de 
contribuição de grupos são organizadas vetorialmente de acordo com a Equação 3.

$$
\begin{aligned}
\theta(i, j)= & \{\theta(1,1), \theta(1,2), \ldots, \theta(1, p)\} \\
& \{\theta(2,1), \theta(2,2), \ldots, \theta(2, p)\} \\
& \{\theta(n, 1), \theta(n, 2), \ldots, \theta(n, p)\} \\
& i=1 \ldots n \\
& j=1 \ldots p
\end{aligned}
$$

onde $j$ representa as propriedades físicas do triglicerídeo e $i$ indica os triglicerídeos. Cada linha do vetor representa um TAG. Esta composição é ajustada de acordo com o procedimento de otimização.

Etapa 3: Inicialização da composição em TAGs

Adota-se composição equimolar nos 35 triglicerídeos.

Etapa 4: Transesterificação do óleo $(T A G>S)$

Os TAGs obtidos na Etapa 3 são transesterificados com metanol para obtenção de ésteres metílicos. A Figura 3 mostra o fluxograma de caracterização composicional construído para caracterização do óleo de microalgas.

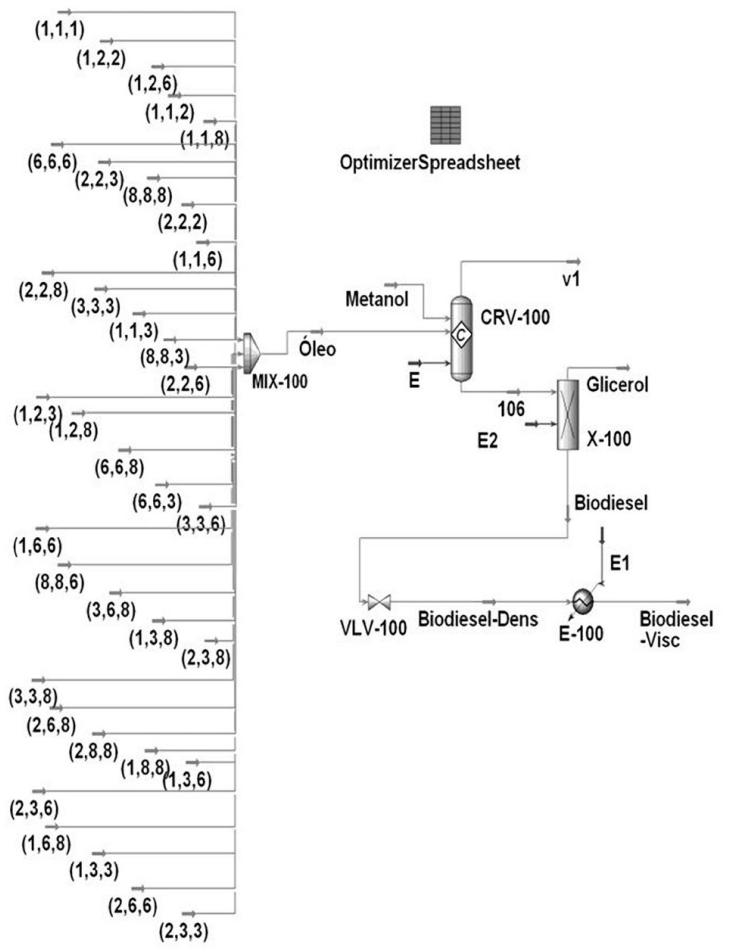

Figura 3. Fluxograma de otimização de formação dos éteres metílicos a partir do óleo de microalga

O fluxograma incorpora um reator de conversão para transesterificação do óleo e a separação do glicerol formado. A transesterificação torna-se necessária posto que os óleos (vegetais e de microalgas) são normalmente reportados na literatura em termos dos ésteres (metílicos) de ácidos graxos obtidos por transesterificação dos TAG's presentes.

Para configurar o cálculo de propriedades termodinâmicas e equilíbrio líquido-vapor no simulador UNISIM Design, utiliza-se: fase vapor via Equação de Estado Peng-Robinson-Twu; fase líquida via Modelo de Solução NRTL com Equação de Antoine para previsão de pressões de vapor de compostos puros (conforme Zhang et al. ${ }^{21}$ ). Para compostos cujos parâmetros de modelos termodinâmicos não estão disponíveis na biblioteca do simulador, métodos internos de contribuição de grupos do próprio software foram utilizados para estimá-los. ${ }^{21}$
O reator de conversão CVR-100 opera a $60{ }^{\circ} \mathrm{C}$ e $400 \mathrm{kPa}$, com uma conversão de $95 \%$ para reação de transesterificação, produzindo biodiesel e glicerol. O separador gravimétrico X-100, a $25^{\circ} \mathrm{C}$, é utilizado para separar o biodiesel formado (100\% de eficiência de separação) para análise das propriedades do biocombustível. Para tal, após o separador, a pressão é reduzida a $100 \mathrm{kPa}$, tornando possível a comparação das densidades do biodiesel de microalga com biodiesel de óleos vegetais. Nesta mesma linha, utiliza-se um aquecedor para aumentar a temperatura da corrente final do biodiesel de 25 para $40{ }^{\circ} \mathrm{C}$, a fim de analisar a viscosidade de acordo com o padrão ASTM.

\section{Etapa 5: Otimização}

A busca da composição do óleo é formulada como um problema de otimização de acordo com as Equações 4 a 6:

$$
\begin{gathered}
\min F(x)=\sum_{I=1}^{N_{A G}} e(x)_{i}^{2} \\
e(x)=C_{R}(x)-C_{O}(x) \\
G(x) \leq 1,19
\end{gathered}
$$

A função objetivo $F(x)$ é definida como a soma dos erros quadráticos da composição molar do biodiesel, $e(x)$. $N_{A G}$ é definido como o número de ácidos graxos, (igual ao número de ésteres metílicos). $\mathrm{O}$ erro $e(x)$ é definido como a diferença entre a composição "real" (i.e., experimental, $C_{R}$ ) de ácidos graxos no óleo da microalga Isochrysis galbana, reportada na Tabela 1, e a composição calculada de ácidos graxos $\left(C_{O}\right)$. O procedimento de otimização é executado no ambiente de simulação UNISIM Design, utilizando o algoritmo BOX, com um máximo de 3,0 x $10^{7}$ iterações. Considera-se que a composição "real" $\left(C_{R}\right)$ de ácidos graxos é igual à de ésteres metílicos formados pela transesterificação dos 35 TAG's adotados na representação composicional do óleo. A otimização submete-se à restrição representada por $G(x)$, a vazão molar de alimentação do processo, adotada igual à reportada por Zhang et al., ${ }^{21}$ com valor de $1,19 \mathrm{kmol} / \mathrm{h}$.

De acordo com o elenco de TAG's proposto para a carga (óleo de microalga Isochrysis galbana), o biodiesel correspondente é constituído por uma mistura de 5 ésteres: M-miristato, M-palmitato, M-oleato, M- estearidonato e M-dodosa-hexaenoato. Para produção de biodiesel, adota-se o fluxograma de processo descrito por Zhang et al. ${ }^{21}$ que utiliza como matéria-prima o óleo de canola, analisado pelos autores como viável para a produção de biodiesel e glicerina de alto grau de pureza. A composição do óleo e as condições experimentais reportadas por Zhang et al.$^{21}$ foram usadas para fins de comparação do desempenho do processo baseado em microalga. Ressaltam-se duas diferenças principais entre esses dois processos: a natureza e composição da carga (óleo de microalga Isochrysis galbana) e o seu impacto na qualidade do produto (biodiesel) e a caracterização composicional - Zhang et al. ${ }^{21}$ abordam de forma simplificada a natureza química da carga, considerando que o óleo de canola é composto exclusivamente por trioleína, produzindo como único éster o metil-oleato. Neste trabalho, foi considerada uma complexa mistura de triglicerídeos (35 TAGs) com composição mássica obtida pelo procedimento de caracterização composicional baseado em rotina computacional de otimização. $\mathrm{O}$ biodiesel obtido difere, pelo rigor da abordagem composicional, em propriedades físico-químicas do biodiesel obtido por aqueles autores, ${ }^{21}$ conforme mostrado na Figura 4.

\section{Estimativa da viscosidade do biodiesel de microalga}

A viscosidade e a densidade do biodiesel são propriedades de extrema importância para avaliar o desempenho dos biocombustíveis em motores. Anand et al. ${ }^{26}$ obtiveram bons resultados para a estimativa da 


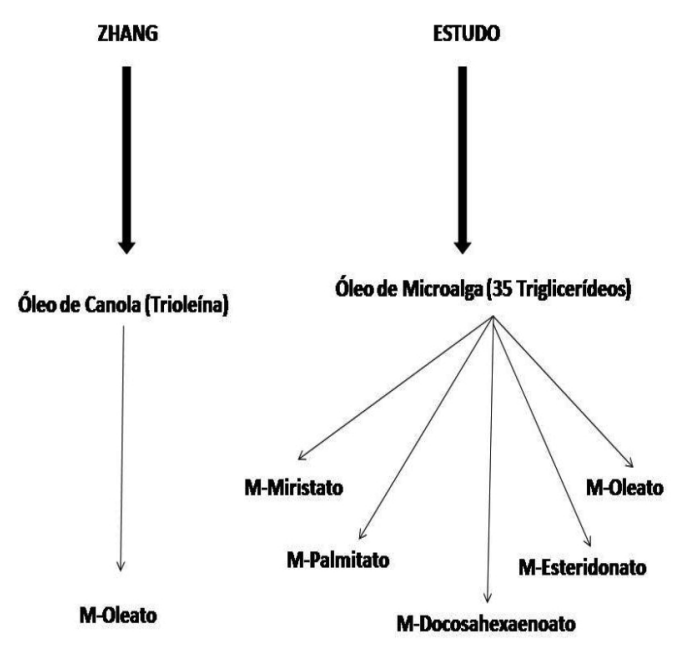

Figura 4. Esquema adotado por Zhang et al. ${ }^{21}$ e o do atual estudo

viscosidade absoluta de óleos vegetais e dos respectivos biocombustíveis empregando a correlação de Sastri-Rao, baseada em contribuição de grupos, no qual a viscosidade da mistura se apresenta em função da composição e da temperatura, conforme com Equações 7 e 8.

$$
\begin{gathered}
\mu=\sum \Delta \eta_{B} p_{v p}{ }^{-(0.25+\alpha)} \\
\ln p_{v p}=\left(4.5398+1.0309 \ln T_{n b}\right)\left\{1-\frac{\left[3-2\left(T / T_{n b}\right)\right]^{\beta}}{T / T_{n b}}\right. \\
\left.-0.38\left[3-2\left(T / T_{n b}\right)\right]^{\beta} \ln \left(T / T_{n b}\right)\right\}^{-0,005} \\
\alpha=\sum \Delta N-0,01 n_{d}-0.0
\end{gathered}
$$

onde $\Delta \eta_{B}$ representa a contribuição de grupo para a obtenção da viscosidade; $p_{v p}$ representa a pressão de vapor das substâncias; o parâmetro $\beta$ é igual a 0,$155 ; \Delta N$ representa a contribuição de grupo para obtenção de $\alpha ; n_{d}$ representa o número de ligações duplas na molécula.

O biodiesel de óleo de microalga difere do biodiesel convencional por seu grau de insaturação. A influência da insaturação no biodiesel é analisada variando-se a composição molar do metil-éster C18:1 (M-oleato) em uma mistura deste com o metil-éster C18:4 (M-estearidonato), que contém 4 insaturações. Adicionalmente, o impacto do peso molecular é avaliado variando-se a composição molar do metil-éster C14:0 (M-meristato) em uma mistura deste com o metil-éster C22:6 (M-dodosa-hexaenoato).

\section{RESULTADOS E DISCUSSÃO}

A metodologia proposta para caracterização composicional de óleo de microalga foi aplicada à espécie Isochrysis galbana, obtendo-se composição mássica (otimizada) dos TAG's reportada na Tabela 4.

O fluxograma de processos proposto por Zhang et al. ${ }^{21}$ para a produção de biodiesel a partir do óleo de canola foi adotado para processamento de óleo de microalga (Figura 5).

Os resultados comparativos dos dois processos e condições de simulação são mostrados na Tabela 5, enquanto as principais propriedades físico-químicas do biodiesel obtido para diferentes matérias-primas (óleos vegetais) e do diesel de petróleo, e para o biodiesel de Isochrysis galbana (estimadas com o simulador UNISIM Design) estão resumidas na Tabela 6. Note-se que não são verificadas
Tabela 4. Composição mássica de TAG's para óleo de microalga Isochrysis galbana

\begin{tabular}{cc}
\hline Triglicerídeos & Fração mássica \\
\hline MMM & 0,072 \\
MMP & 0,001 \\
MMO & 0,020 \\
MMS & 0,062 \\
MMD & 0,073 \\
MPP & 0,051 \\
MPO & 0,006 \\
MPS & 0,014 \\
MPD & 0,004 \\
MOO & 0,062 \\
MOS & 0,019 \\
MOD & 0,007 \\
MSS & 0,022 \\
MSD & 0,006 \\
MDD & 0,019 \\
PPP & 0,085 \\
PPO & 0,071 \\
PPS & 0,032 \\
PPD & 0,000 \\
POO & 0,003 \\
POS & 0,009 \\
PSD & 0,042 \\
PSS & 0,044 \\
POD & 0,100 \\
PDD & 0,008 \\
OOO & 0,013 \\
OOS & 0,034 \\
OOD & 0,004 \\
OSD & 0,035 \\
SSD & 0,001 \\
SSS & 0,017 \\
SSO & 0,035 \\
DDO & 0,012 \\
DDS & 0,006 \\
DDD & 015 \\
\hline &
\end{tabular}

diferenças significativas tanto no processo (Tabela 5) quanto no produto (Tabela 6), para óleo de canola e de microalga. Verifica-se uma razoável coerência nos valores calculados para o biodiesel de óleo de microalga, que encontra respaldo no fato da sua composição em ácidos graxos ser semelhante à exibida pela maioria dos óleos vegetais. Adicionalmente, observa-se que o valor da viscosidade do biodiesel de óleo de microalga pela correlação de Sastri-Rao modificada se apresenta em conformidade com os valores reais encontrados para maioria dos óleos vegetais (Tabela 6).

As semelhanças observadas entre o biodiesel de óleos vegetais e de óleo de microalga permitem concluir que o último se apresenta de fato como uma matéria-prima alternativa, posto que com os mesmos equipamentos de processo e condições de operação similares foi possível atender às especificações do produto. Considerando-se que não há relato de características de biodiesel de microalga em escala industrial, que a metodologia se baseia em uma descrição composicional de razoável fidelidade quando comparada a estudos reportados na literatura (e.g., Zhang et al. $^{21}$ ) e que as condições e escala do processo simulado são compatíveis com produção industrial, a conclusão alcançada deve ser destacada. 


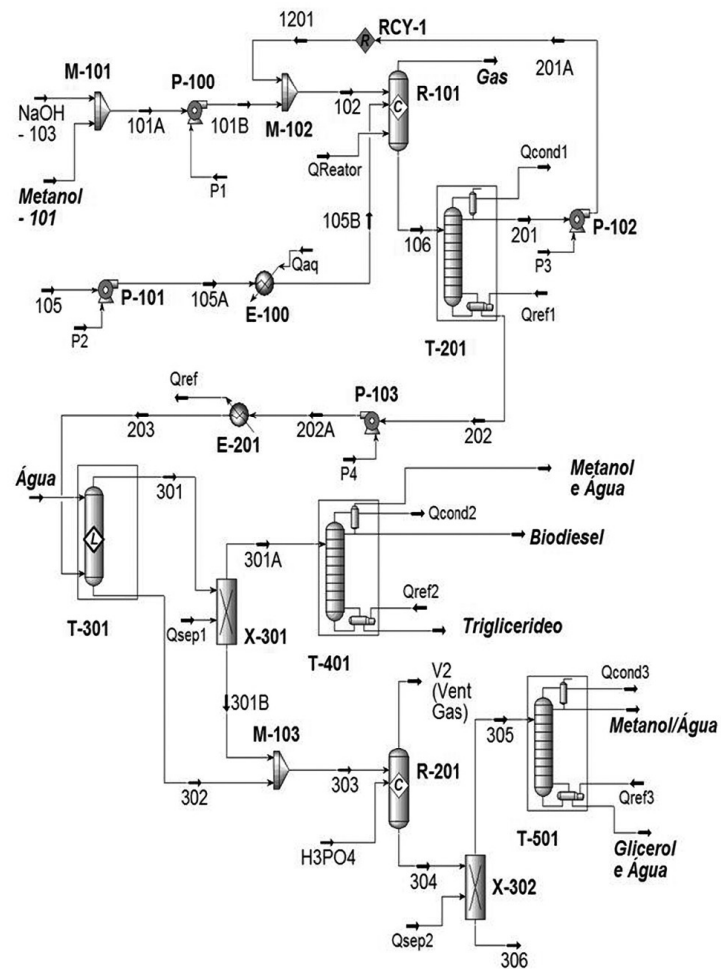

Figura 5. Fluxograma do processo utilizado no trabalho

Apresentam-se na Tabela 7 as propriedades do biodiesel de Isochrysis galbana (obtidos neste trabalho) com o biodiesel da espécie Chlorella protothecoides, o diesel fóssil e a especificação da ANP. ${ }^{27,28}$

Por último, a Figura 6 apresenta a variação da viscosidade, em função da concentração e grau de insaturação, para duas misturas binárias: (a) M-oleato e M- estearidonato; (b) M-meristato e M-dodosa-hexaenoato. Observa-se que o peso molecular exerce maior influência na viscosidade do biodiesel quando comparada à do grau de insaturação de metil-ésteres. Nota-se, também, que as insaturações e o peso molecular causam variações significativas na densidade.

\section{CONCLUSÃO}

Neste trabalho, apresenta-se uma metodologia de caracterização composicional de óleos aplicada ao óleo da microalga Isochrysis galbana. A descrição composicional envolve um amplo elenco de
Tabela 5. Condições de simulação e resultados comparativos

\begin{tabular}{|c|c|c|}
\hline & Canola $^{21}$ & Microalga \\
\hline \multicolumn{3}{|c|}{ COLUNA T-201 (Recuperação de Metanol) } \\
\hline $\mathrm{N}^{\mathrm{o}}$ de estágios & 5 & 5 \\
\hline Razão de refluxo & 2 & 2 \\
\hline Eficiência de separação & $94,0 \%$ & $93,6 \%$ \\
\hline \multicolumn{3}{|l|}{ E-201 } \\
\hline Temperatura de Saída & $60^{\circ} \mathrm{C}$ & $60^{\circ} \mathrm{C}$ \\
\hline \multicolumn{3}{|l|}{ COLUNA T-401 } \\
\hline $\mathrm{N}^{\circ}$ de estágios & 4 & 4 \\
\hline Razão de refluxo & 2 & 2 \\
\hline \multicolumn{3}{|l|}{ Corrente Biodiesel } \\
\hline Temperatura & $193,7^{\circ} \mathrm{C}$ & $179^{\circ} \mathrm{C}$ \\
\hline Pressão & $10 \mathrm{kPa}$ & $10 \mathrm{kPa}$ \\
\hline Grau de pureza & $99,65 \%$ & $99,97 \%$ \\
\hline \multicolumn{3}{|c|}{ COLUNA T-301 (Lavagem do biodiesel) } \\
\hline $\mathrm{N}^{\circ}$ de estágios & 4 & 4 \\
\hline Temperatura de entrada da água & $25^{\circ} \mathrm{C}$ & $25^{\circ} \mathrm{C}$ \\
\hline \multicolumn{3}{|l|}{ Corrente 301A } \\
\hline Óleo & $<6 \%$ & $4,99 \%$ \\
\hline Metanol & & $0,18 \%$ \\
\hline Água & & $0,02 \%$ \\
\hline \multicolumn{3}{|l|}{ Corrente 303} \\
\hline Glicerol & $81 \%$ & $79,68 \%$ \\
\hline $\mathrm{NaOH}$ & $9 \%$ & $7,65 \%$ \\
\hline Água & $8 \%$ & $8,25 \%$ \\
\hline Metanol & $3 \%$ & $4,42 \%$ \\
\hline \multicolumn{3}{|c|}{ COLUNA T-501 (Purificação de Glicerol) } \\
\hline $\mathrm{N}^{\circ}$ de estágios & 4 & 4 \\
\hline Razão de refluxo & 2 & 2 \\
\hline \multicolumn{3}{|l|}{ Corrente Glicerol e Água } \\
\hline Glicerol & $92 \%$ & $90,34 \%$ \\
\hline
\end{tabular}

TAGs, o que distingue o estudo de abordagens anteriores, normalmente simplificadas para incluir um único ácido graxo e o respectivo éster metílico. ${ }^{21} \mathrm{O}$ procedimento emprega simulação rigorosa (UNISIM Design), o que permite calcular propriedades caracterizadoras de biodiesel, verificando-se a adequação destas especificações

Tabela 6. Comparação das propriedades de biodiesel da microalga I. galbana e biodiesel de canola obtidas neste trabalho, de biodieseis de óleos vegetais e de óleo diesel

\begin{tabular}{|c|c|c|c|c|c|c|c|}
\hline \multirow{2}{*}{ Propriedades } & \multicolumn{5}{|c|}{ Biodiesel de óleos vegetais } & \multirow{2}{*}{ Microalga I.g (e) } & \multirow{2}{*}{ Óleo diesel ${ }^{(f)}$} \\
\hline & Mamona $^{(\mathrm{f})}$ & Babaçu $u^{(f)}$ & Dendê $\hat{(f)}^{(\mathrm{f})}$ & Soja $^{(f)}$ & Canola & & \\
\hline Densidade a $25^{\circ} \mathrm{C}\left(\mathrm{g} / \mathrm{cm}^{3}\right)(\mathrm{a})$ & 0,9190 & 0,8865 & 0,8597 & 0,8750 & 0,8713 & 0,8801 & $0,8497^{\text {(a) }}$ \\
\hline Viscosidade a $37,8^{\circ} \mathrm{C}(\mathrm{cSt})$ & 21,6 & 3,9 & 6,4 & 6,0 & 4,53 & 3,76 & 3,04 \\
\hline Poder calorífico (MJ/kg) & 37,87 & 39,52 & 39,9 & 39,86 & nd & 31,0 & 45,85 \\
\hline Ponto de névoa $\left({ }^{\circ} \mathrm{C}\right)$ & -6 & -6 & 6 & nd & nd & nd & 1 \\
\hline Índice de cetano & nd & 65 & nd & 57,5 & nd & 45,99 & 45,8 \\
\hline Inflamabilidade $\left({ }^{\circ} \mathrm{C}\right)$ & 208 & nd & nd & 184 & nd & $62,09^{(\mathrm{c})}$ & 55 \\
\hline Ponto de fluidez $\left({ }^{\circ} \mathrm{C}\right)$ & -30 & nd & nd & -3 & nd & 7,82 & nd \\
\hline Destilação a $50 \%\left({ }^{\circ} \mathrm{C}\right)$ & 301 & 291 & 333 & 340 & nd & $315,6^{(\mathrm{b})}$ & 278 \\
\hline Destilação a $90 \%\left({ }^{\circ} \mathrm{C}\right)$ & 318 & 333 & 338 & 342 & nd & $351,5^{(b)}$ & 373 \\
\hline
\end{tabular}

(a) Densidade a $20^{\circ} \mathrm{C}$, (b) ASTM D86, (c) ASTM D97, (d) Ref. 21, (e) Isochrysis galbana, (f) Ref. 27; nd = não determinado 
Tabela 7. Comparação das propriedades do biodiesel de Isochrysis galbana, Chlorella protothecoides, diesel fóssil e especificação ANP

\begin{tabular}{|c|c|c|c|c|}
\hline \multirow{2}{*}{ Propriedades } & \multicolumn{2}{|c|}{ Biodiesel de microalgas } & \multirow{2}{*}{ Diesel fóssil ${ }^{\mathrm{c}}$} & \multirow{2}{*}{$\begin{array}{c}\text { Resolução ANP } \\
n^{\circ} 42 / 042^{\mathrm{d}}\end{array}$} \\
\hline & I. $g^{\mathrm{a}}$ & Ch.p. ${ }^{\mathrm{b}}$ & & \\
\hline Densidade $\left(\mathrm{kg} \mathrm{L}^{-1} \mathrm{a} 20^{\circ} \mathrm{C}\right)$ & 0,8801 & 0,864 & 0,838 & $0,820-0,865$ \\
\hline Viscosidade (cSt a $\left.40^{\circ} \mathrm{C}\right)$ & 3,76 & 5,2 & $1,9-4,1$ & 2,0 a 5,0 \\
\hline Ponto de fulgor $\left({ }^{\circ} \mathrm{C}\right)$ & ns & 115 & 75 & Min 100 \\
\hline Ponto de solidificação $\left({ }^{\circ} \mathrm{C}\right)$ & ns & -12 & -50 a 10 & nd \\
\hline Ponto de entupimento de filtro a frio $\left({ }^{\circ} \mathrm{C}\right)$ & ns & -11 & $-3,0(\max -6,7)$ & - \\
\hline Índice de acidez (mg KOH g $\left.{ }^{-1}\right)$ & ns & 0,374 & Max 0,5 & Max 0,8 \\
\hline Poder calorífico $\left(\mathrm{MJ} \mathrm{kg}^{-1}\right)$ & 39 & 39 & $40-45$ & nd \\
\hline Razão H/C & 1,79 & nd & 1,81 & nd \\
\hline
\end{tabular}

(a) Isochrysis galbana; (b) Chlorella protothecoides, Ref. 19; (c) Ref. 19; (d) Ref. 28; ns - não simulado; nd- não disponível
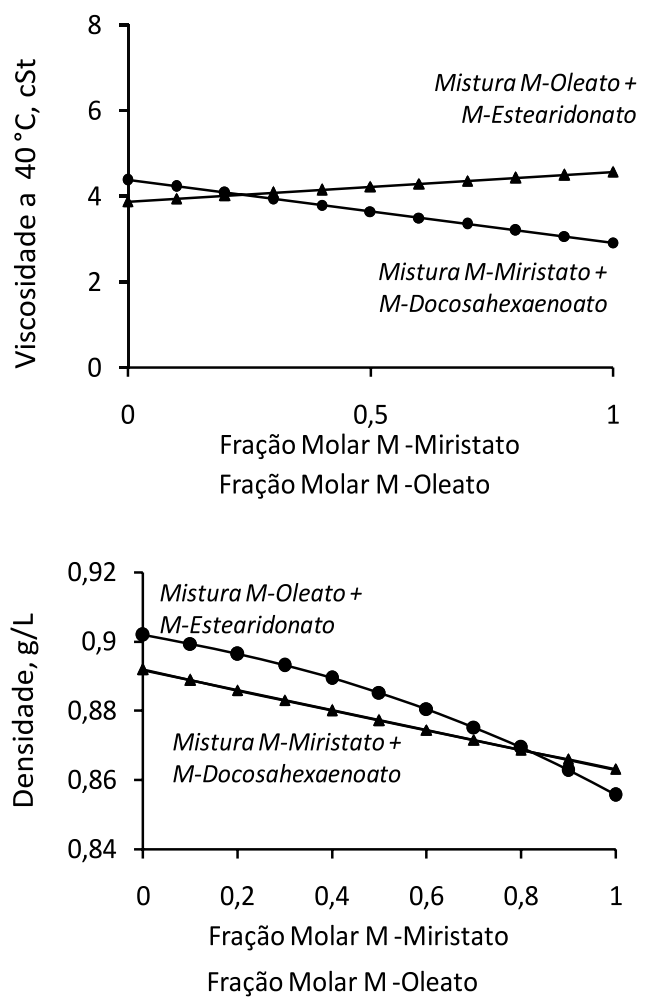

Figura 6. Viscosidade e densidade versus fração molar para duas misturas: (p) metil-éster C18:1 (M-oleato) + metil-éster C18:4 (M-estearidonato); ( ) metil-éster C14:0 (M-meristato $)+$ metil-éster C22:6(M-dodosa-hexaenoato)

(e.g., ASTM). Adicionalmente, as propriedades calculadas para o biodiesel e o óleo de microalgas mostraram-se adequadas quando comparadas com valores reportados na literatura ${ }^{27}$ para biodieseis de óleos vegetais. A caracterização composicional obtida é empregada em simulação de fluxograma de processo de produção de biodiesel proposto por Zhang et al., ${ }^{21}$ obtendo-se características semelhantes para os dois biodieseis, concluindo-se que o óleo de microalga é uma matéria-prima tecnicamente viável, pois foi possível atender às especificações do produto com mesma configuração de processo. Destaca-se que não se observam alterações significativas nas condições de operação quando comparado ao processo baseado em óleo de canola, ${ }^{21}$ concluindo-se pela viabilidade técnica do uso desta matéria-prima para produção de biodiesel. Considerando-se que não há relato na literatura de características de biodiesel de microalga em escala industrial, que a metodologia se baseia em uma descrição composicional de razoável fidelidade se comparada a estudos reportados na literatura, ${ }^{21}$ e que as condições e escala do processo simulado são compatíveis com produção industrial, a conclusão alcançada deve ser destacada. Ressalta-se que o biodiesel de microalgas tem como vantagem alta produtividade (L/ha) e o fato de não competir com alimentos por terras aráveis. Contudo, a viabilidade econômica da produção deste biodiesel ainda deve ser avaliada.

\section{REFERÊNCIAS}

1. Clark, J. Em Handbook of Green Chemistry \& Technology; Clark, J.; Macquarrie, D., eds.; Blackwell Science: Oxford, 2002, cap. 2.

2. Rodolfi, L.; Zittelli, G. C.; Bassi, N.; Padovani, G.; Biondi, N.; Bonini, G.; Tredici, M. R; Biotechnol. Bioeng. 2009, 102, 100.

3. Huang, G.; Chen, F.; Wei, D.; Zhang, X.; Chen, G.; Appl. Energy 2009, 6.

4. Gouveia, L.; Oliveira, A. C.; J. Ind. Microbiol. Biotechnol. 2009, 36, 269.

5. Mata, T.; Martins, A. A.; Caetano, N. S.; Renewable and Sustainable Energy Reviews 2010, 14, 217.

6. Carioca, J. O. Em Química verde no Brasil: 2010 - 2030; Carioca, J. O.; Almeida, M. F.; Seidl, P. R., eds.; Centro de Gestão e Estudos Estratégicos: Brasília, 2010, cap. 1.

7. http://www.sae.gov.br, acessada em Junho 2011.

8. Brennan, L.; Owende, P.; Renewable e Sustainable Energy Reviews 2010, 14, 557.

9. http://ec.europa.eu/energy/energy_policy/doc/01_energy_policy_for_ europe_en.pdf, acessada em Março 2011.

10. http://www.iea.org/weo/2007.asp, acessada em Março 2011.

11. Wang, B.; Li, Y.; Wu, N.; Lan, C. Q.; Appl. Microbiol. Biotechnol. 2008, $79,707$.

12. Borges, L.; Faria, B. M.; Odebrecht, C.; Abreu, P. C.; Atlântica 2007, $29,35$.

13. Monteiro, J.; Tese de Mestrado, Universidade Federal do Rio de Janeiro, Brasil, 2009.

14. Madigan, M. T. Em Brock Biology of Microrganisms; Madigan, M. T.; Martinko, J. M.; Parker, J., eds.; Prentice-Hall International: Englewood Cliffs, 1996.

15. Tortora, G. J. Em Microbiologia; Tortora, G. J.; Funke, B. R.; Case, C. L., eds.; ArtMed: Porto Alegre, 2005.

16. Buchgraber, M.; Ulberth, F.; Emons, H.; Anklam, E.; Eur. J. Lipid Sci. Technol. 2004, 106, 621.

17. Rinaldi, R.; Garcia, C.; Ledo, M.; Rossi, A.; Quim. Nova 2007, 30, 1374.

18. Penteado, D.; Tese de Mestrado, Universidade Federal do Paraná, Brasil, 2010.

19. Miao, X.; Wu, Q.; Bioresour. Technol. 2006, 97, 841. 
20. Belardi, H.; Grima, M.; Chisti, Y.; Enzyme Microb. Technol. 2000, 26, 516.

21. Zhang, Y.; Dub, M.; MClean, D.; Kates, M.; Bioresour. Technol. 2003, 89,1 .

22. Lin, Y.; Chang, F.; Tsao, C.; Leu, J.; Biochem. Eng. J. 2007, 37, 166.

23. Constantinou, L.; Gani, R.; O’Connell, J.; Fluid Phase Equilib. 1995, $103,11$.

24. Marrero, J.; Gani, R.; Fluid Phase Equilib. 2001, 183, 183.
25. Poling, B. E. Em The Properties of Gases e Liquids; Poling, B. E.; Prausnitz, J. M.; O’Connell, J. P., eds.; McGraw-Hill: New York, 2004. 26. Anand, K.; Ranjan, A.; Mehta, P.; Energy Fuels 2009, 24, 664.

27. Costa, P. R.; Rossi, L. F.; Zagonel, G. F.; Ramos, L. P.; Quim. Nova 2000, 23, 531 .

28. http://www.anp.gov.br, acessada em Março 2011. 